\title{
A Novel Algorithm for Human Fall Detection using Height, Velocity and Position of the Subject from Depth Maps
}

\author{
Yoosuf Nizam $^{1}$, M. Mahadi Abdul Jamil,", Mohd Norzali Haji Mohd², M. \\ Youseffi ${ }^{3}$, M. C. T. Denyer ${ }^{4}$
}

\author{
${ }^{1}$ Biomedical Engineering Modeling and Simulation (BIOMEMS) Research Group, Faculty of Electrical and Electronic \\ Engineering, Universiti Tun Hussein Onn Malaysia, 86400 Parit Raja, Batu Pahat, Johor, Malaysia. \\ ${ }^{1}$ Embedded Computing Systems (EmbCos), Faculty of Electrical and Electronic Engineering, Universiti Tun Hussein \\ Onn Malaysia, 86400 Parit Raja, Batu Pahat, Johor, Malaysia. \\ ${ }^{1}$ Faculty of Engineering and Informatics, School of Engineering, University of Bradford, Bradford, West Yorkshire \\ BD7 1DP, UK. \\ ${ }^{1}$ School of Medical Sciences, University of Bradford, Bradford, West Yorkshire BD7 1DP, UK.
}

Received 9 January 2018; accepted 16 May 2018, available online 2 July 2018

\begin{abstract}
Human fall detection systems play an important role in our daily life, because falls are the main obstacle for elderly people to live independently and it is also a major health concern due to aging population. Different approaches are used to develop human fall detection systems for elderly and people with special needs. The three basic approaches include some sort of wearable devices, ambient based devices or non-invasive vision-based devices using live cameras. Most of such systems are either based on wearable or ambient sensor which is very often rejected by users due to the high false alarm and difficulties in carrying them during their daily life activities. This paper proposes a fall detection system based on the height, velocity and position of the subject using depth information from Microsoft Kinect sensor. Classification of human fall from other activities of daily life is accomplished using height and velocity of the subject extracted from the depth information. Finally position of the subject is identified for fall confirmation. From the experimental results, the proposed system was able to achieve an average accuracy of $94.81 \%$ with sensitivity of $100 \%$ and specificity of $93.33 \%$.
\end{abstract}

Keywords: Fall detection, Kinect sensor, Depth images, Non-invasive, Depth sensor

\section{Introduction}

Classification of daily activities of human life is a key topic in the field of assistive technology due to emergence of numerous applications requiring human posture and movement recognition. Among such application is the human fall detection system, which also requires distinguishing human activities. Human fall detection systems are very often required for many people in today's aging population including elderly and people with special needs such as disable and obese. It is also a major health concern to many communities in todays' aging population, because human falls are the main causes of injury related deaths for seniors aged 79 and above $([1,2]$. Injuries from fall is also a major public health concern [3] and it is the main obstacle for elderly people to live independently.

Fall detection systems use various approaches to distinguish human fall from other activities of daily life, such as wearable based sensors, non-wearable sensors and vision-based sensors. Thus, the three basic approaches used in developing human fall detection systems are wearable based device, camera (vision) based and ambience-based devices. Wearable based devices such as fall detection belts and ambient devices using vibration and pressure sensors are used to generate high false alarms. As a result, such devices are often rejected by the users. On the other hand, vision-based fall detection systems are very accurate in classifying human falls. Therefore, vision-based sensors [4-6] for human detection and identification are important sensors among the researchers, especially as they tend to base their fall detection on non-wearable sensors [7].

Vision based approaches using live cameras are accurate in detecting human falls and it does not generate high false alarms. However normal cameras require adequate lighting and it does not preserve the privacy of users. As a result, users are not willing to accept having such systems installed at their home. Moreover, with normal color camera it is not possible to achieve the accuracy than that of a depth sensor, in extracting the position of the subject. Thus, a depth sensor would be a preferable option over color camera, since it can identify human even in dark and at the same time preserve the privacy of users and it has shown good performance in previous works [8-12]. One of the sensor that generate depth images to track human skeleton is Microsoft Kinect for windows. This paper proposes a vision-based fall detection system, which uses depth information from Kinect sensor to classify human fall from other activities 
of daily life. The algorithm will first classify human activities using height and velocity of the subject to identify any potential fall movement. If any such movement is detected the system will extract the position of the subject to confirm fall.

\section{Related works}

Since this paper focuses on the use of the depth information to categorize human activities for fall detection, this section will review only a set of selected papers that had based their fall detection on depth sensors.

Combination of a wearable wireless accelerometer and a depth sensor-based fall detection were conducted in which used distance between the person centre of gravity and floor to confirm fall. The authentication of fall after potential fall indication from the accelerometer is accomplished from a Kinect sensor depth images [13]. Another study proposed fall detection using ceiling mount depth camera [14].

A statistical method for human fall detection based on how human move during the last few frames is proposed by Zhang et at. They used statistical decision making as opposed to hard-coded decision making used in related works. Duration of fall in frames, total head drop-change of head height, maximum speed (largest drop in head height), smallest head height and fraction of frames where head-drop is considered for fall detection [15].

A Spatio temporal context (STC) tracking of depth images from a Kinect sensor was proposed by Yang et al, where the parameters of the Single Gauss Model (SGM) are estimated and the coefficients of the floor plane are extracted in pre-processing. Than Foreground coefficient of ellipses is used to determine the head position and STC algorithm is used to track head position. The distance from head to floor plane is calculated in every following frame and a fall is indicated if an adaptive threshold has reached [16].

Bian et al presented a method for fall detection based on two features: distance between human skeleton joints and the floor, and the joint velocity. A fall is detected if the distance between the joints and the floor is close. Then the velocity of the joint hitting the floor is used to distinguish the event from a fall accident or a sitting/lying down on the floor [7].

A fall detection and reporting system using Microsoft Kinect sensor presented by Kawatsu et al, uses two algorithms. The first uses only a single frame to determine a fall and the second uses time series data to distinguish between fall and slow lying down on the floor. For these algorithms they use the joint position and the joint velocities. The reporting can be sent as emails or text messages and can include pictures during $\&$ after the fall [17].

In another study, a mobile robot system is introduced, which follows a person and detects when the person has fallen using a Kinect sensor. They used the distance between the body joints and the floor plane to detect fall [18].
Rougier et al presented a method for fall detection that uses human centroid height relative to the ground and body velocity. They have also dealt with occlusions, which was a weakness of previous works and claimed to have a good fall detection results with an overall success rate of $98.7 \%$ [19].

A privacy-preserving fall detection method for indoor environment using Microsoft Kinect depth sensor was proposed by Gasparrini et at. They used Ad-Hoc segmentation algorithm on a ceiling mount configuration. The raw data directly from the sensor were analyzed and the system extracts the elements to classify all the blobs in the scene through the implemented solutions. The recognized human subjects from the blobs is followed by a tracking algorithm between frames. A fall is detected if the depth blob associated to a person is near to the floor [20].

They proposed another work using a wearable device along with the depth sensor using three algorithms. The first algorithm uses a wearable accelerometer at wrist and Kinect sensor. For the second algorithms, the wearable accelerometer is place at waist. The third algorithm avoids using the orientation of the accelerometer and uses the distance of spine joint to floor instead of the angle of the accelerometer which is orientation of the sensor [21].

The related works either uses height of subject and the velocity of the joints or position and velocity of joints to classify human falls [22]. They used Different algorithms to extract the subject from the depth images. The proposed algorithm uses combination velocity of body and height of subject to classify human fall from other activities of daily life.

\section{Methodology}

The method proposed in this paper employs the height of the subject and velocity of the body to identify any potential fall movements. Once such a movement is observed the position of the joints (human joints) is measured with respective to the floor plan to confirm if the subject is lying on the floor. The algorithm starts with the extraction of depth information from Kinect v1 sensor for the extraction of 3D human joint position and floor plane. These data are then used to compute the velocity of the body, the distance between the head to floor plane (height) and the position of the other joints to classify an unintentional human fall from other activities of daily life

Overview of the system: Human skeleton coordinates generated from the Microsoft Kinect Sensor are used in the proposed algorithm for fall detection. The skeleton joints data are stored as $(\mathrm{x}, \mathrm{y}, \mathrm{z})$ coordinates expressed in meters. In this coordinate system, the positive y-axis extends vertical upwards from the depth sensor, the positive $\mathrm{x}$-axis extends to the left placing the Kinect sensor on a surface level and the positive $\mathrm{z}$-axis extending in the direction in which the Kinect is pointed. The $\mathrm{z}$ value gives the distance of the object to the sensor (objects close to sensor will have a small depth value and object far away will have larger depth value). Using these joint coordinate data, the movement of any joint, velocity 
and position of the subject can be computed along with the direction respective to the previous joint position. The following Fig. 1 displays the skeleton generated from the sensor, labeled with joints considered in the algorithm. Table 1 shows the combinations of joints considered for different parameters used in the proposed algorithm for the classification process of human fall from other activities of daily life.

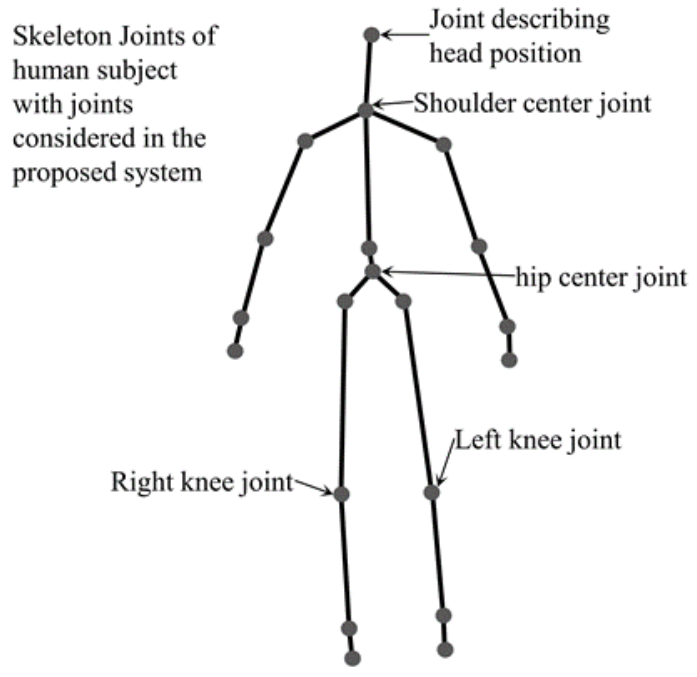

Fig. 1 Illustration of joints considered in the proposed algorithm.

Combinations of labeled joints in Fig. 1. are used for the calculations of subject's height, velocity, speed and position (posture) as described in Table 1. The proposed fall detection algorithm using these parameters are discussed in the next section and the detailed derivation of the parameters in the following section. In the following Table 1, the tick mark indicates that the joint is used for the calculation of specific parameter.

Table 1 Joint coordinates used for the different parameters.

\begin{tabular}{lllll}
\hline Joints & Head & $\begin{array}{l}\text { Shoulder } \\
\text { center }\end{array}$ & $\begin{array}{l}\text { Hip } \\
\text { center }\end{array}$ & $\begin{array}{l}\text { Left and } \\
\text { right Knee }\end{array}$ \\
\hline Height & $\checkmark$ & Not used & Not used & $\checkmark$ \\
Velocity & $\checkmark$ & $\checkmark$ & $\checkmark$ & Not used \\
Speed & Not used & $\checkmark$ & Not used & Not used \\
Position & $\checkmark$ & $\checkmark$ & $\checkmark$ & $\checkmark$ \\
\hline
\end{tabular}

Fall detection algorithm: The proposed algorithm for fall detection goes through four stages which starts after a person is detected in the acquired depth image from the sensor. If a person is not detected the loop will go back and acquire the next image. If a person is detected stage 1 will be executed, where the algorithms will extract skeleton coordinates data of all joints and take a delay of two frames to extract another set of skeleton data than calculate the initial velocity and height of the subject. The process will skip 10 frames before calculating the next velocity for the identification of any abnormal velocity with respective to the previously calculated velocity. If it senses an abnormal increase in velocity the next stage will calculate the height of the subject to identify the direction of the movement or change with respective to the previously calculated height. This is because an abnormal increase in velocity can be caused from many activities where the pattern and the changes in direction of height are different like increase in walking speed (increased step frequency or step length), sitting on chair or floor, lying down on floor or bed and running. For an example for walking and running the height changes will be a straight fluctuation on to any direction and for other activities mentioned above the direction will be straight down or most probably diagonally down to y-axis of the image. Therefore, the system can differentiate the activities by considering how far the height drops and the position of joints (posture) after the change (abnormal velocity) stabilizes. This process of classifying human fall from other activities of daily is illustrated in Fig. 2. As seen from the Fig. 2., the algorithm will execute stage 3 if it couldn't sense any abnormal change in velocity (from stage 1) or in height (from stage 2), where the system will check for any activity or movement. If movements are detected than the system will display the joint coordinates and start the process all over. If no activity is detected in stage 3 or unusual height change is observed in stage 2, the process will activate the microphone array of the sensor to listen to any voice from a falling person and then system will execute stage 4 after skipping 15 frames (half of a second). In stage 4, the system will compute the joint position to predict the posture and the algorithm will check if the joints are below the previously extracted knee joints (left knee and right knee) in stage 1. This is because for activities like lying on bed the changes of velocity and height follow similar like during a fall except that, at the end the subject will be lying on bed. During a fall, all the joint height should be below or equal to the knee joint before the detection of abnormal change in velocity or height. For lying on bed, the joint heights will probably be above the previous knee joint height because the subject will be lying on bed. If the joints are below the previous knee joint position than the system will confirm it as a fall. This is because a lying posture of joints can be observed from activities other than fall like lying on bed or even fall on bed where the consequences will not be severe.

Some of the daily activities that are closely related to human fall are shown in Table 2 along with the characteristics considered in the classification of the events 
Table 2 Characteristics of some movements.

\begin{tabular}{ll}
\hline Activity & Characteristics \\
\hline Sitting on chair & Hip center and head vertical, angle formed from hip center to head \& hip center to knee $<90^{\circ}$ \\
Sitting on floor & Hip center and head vertical, hip center, left and right knee on floor \\
Lying on floor & Hip center and head horizontal, all joints below or equal previous knee height \\
Lying on bed & Hip center and head horizontal, all joints above or equal to previous knee height \\
Fall & Velocity \& height is high, all joints below or equal previous knee height \\
\hline
\end{tabular}

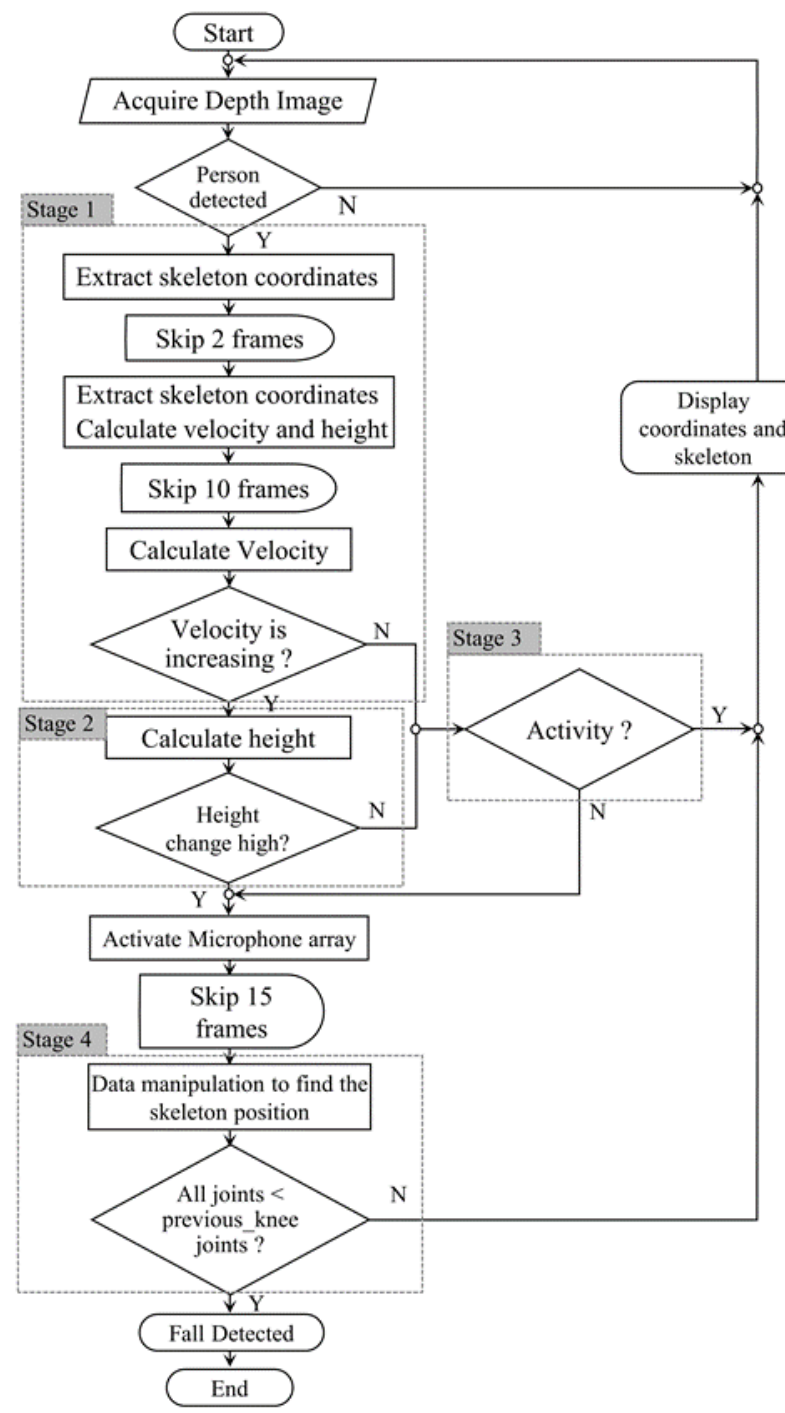

Fig. 2 Process flow of the proposed algorithm.

Floor Plane and calculation of other parameters: This section will discuss on the calculation of the parameters used in the proposed algorithm including height, velocity, speed and position of joints. Height is typically referred to the distance from floor to head (height of the subject) but in an instance in stage 4 , the distance from knee joints (left knee and right knee) to floor (knee joint height) is also considered for confirming fall which is computed stage 1. Speed is the rate of change of position of the subject, calculated from the change of position of shoulder center over time. Velocity is the change of position per unit time in a particular direction. In other words, speed is the magnitude component of the velocity and velocity contains speed (magnitude component) plus direction component. In this paper, speed is used to calculate the speed of walking and running. Velocity is used to classify different activities in terms of rate of change of head, shoulder center and hip center with direction. The position of joints is computed by considering the height and the angles in between the joints as per the characteristics mentioned in Table 2 for the joints in Table 1.

To calculate the height of the subject and the distance from knee joints to floor, the algorithm uses the floor plan provided by the Kinect and the generated skeleton coordinates as a basis for the distance calculation in equation 2. To find out the floor plane equation at start up, three points from 3D floor plane is chosen and then the system automatically solve the floor plane equation. The skeleton frame generated from depth image also contains floor-clipping-plane vector, which has the coefficients of an estimated floor-plane equation as shown by Equation (1).

$$
\mathrm{Ax}+\mathrm{By}+\mathrm{Cz}+\mathrm{D}=0
$$

$$
\begin{aligned}
& \text { Here: A = vFloorClipPlane.x } \\
& \text { B }=\text { vFloorClipPlane.y } \\
& \text { C }=\text { vFloorClipPlane.z } \\
& \text { D }=\text { vFloorClipPlane.w }
\end{aligned}
$$

The equation is normalized such that $\mathrm{D}$ is the height of the camera from the floor in meters. Using this equation, the floor plane or even stair plane can be detected at the same time. To calculate the distance between any joint and the floor, the joint coordinates and floor plane equation can be applied to the following Equation (2).

$$
\mathrm{D}(\text { distance }- \text { joint to floor })=\frac{|A x+B y+C z+D|}{\sqrt{\left(A^{2}+B^{2}+C^{2}\right)}}
$$

Where: $\mathrm{x}, \mathrm{y}, \mathrm{z}$ are the coordinates of the joint.

For speed and velocity calculation, joint coordinates are extracted after every consecutive frame. Speed (of walking or running) is calculated by taking the difference of the shoulder center position in between three frames (skipping one frame) from the respective axis after considering the direction of the movement (direction component of velocity). The direction is obtained using the coordinate system of the Kinect. As per the coordinate 
system shown in Fig. 4, any movement to the right or top or going far (to any axis) gives a positive value for the distance difference from current position to previous position. Similarly, any movement to left or down or coming close gives a negative value for the distance difference from current position to previous position. Using this concept, the direction is determined to all the movements shown in Fig. 4. If the direction is straight (right or left), x-axis is considered and if it is horizontal (up or down) $y$-axis and z-axis is used if the movement is coming close or going far as shown in Fig. 3a to $3 \mathrm{c}$ respectively.

Once the difference of shoulder center position is determined after considering the direction of the movement, it is than divided by the time taken for the movement to calculate the speed as shown in equation 3 . The time taken for the movement is $1 / 15$ seconds, because the sensor generates 30 frames per second and the joint position is taken after skipping one frame (time for two frames). For the magnitude component of velocities, the same concept as for speed (equation 3 ) is used except that the speed for the shoulder center and hip center is also calculated together with direction.

$$
\text { Speed }=\frac{D_{c}-D_{p}}{t_{c}-t_{p}} \text { Meter/second }
$$

Where Dc is the Current Distance (current joint coordinate), Dp is the Previous Distance (previous joint coordinate), tc is the current time in second and tp is the previous time in second.

If the direction is vertically (irregular) to any side (any axis), the distance travelled, cannot be simple calculated by subtracting the position between two frames on any axis, because the changes is not on the axis and so if the changes is considered as on the axis, then the distance will be less than the actual distance travelled. This distance can be calculated by assuming the distance travelled as the hypotenuse of a right-angled triangle whose opposite and adjacent are form by joining the current and previous position of the particular joint to the $\mathrm{x}$-axis and $\mathrm{y}$-axis. Here the actual distance travelled is the hypotenuse and the other two sides (opposite and adjacent) of the triangle will be formed by taking the straight movement to the two axes ( $\mathrm{x}$ and $\mathrm{y}$ ) depending on the direction of the movement. For an example if the direction is diagonally down or up with respective to yaxis than the opposite and adjacent of the triangle will be formed from movement straight onto $\mathrm{x}$-axis and $\mathrm{y}$-axis as shown in Fig. 3d. The formula used to calculate this distance (hypotenuse of the triangle formed) is shown in equation 4. Once distance is calculated the magnitude part of the velocity is calculated by using the equation 3 .

$$
\begin{aligned}
& \mathrm{D}(\text { distance change for irregular movements })= \\
& \sqrt{\left(\left(\mathrm{y}-\mathrm{y}^{\mathrm{x}}\right)^{2}+\left(\mathrm{x}-\mathrm{x}^{\mathrm{x}}\right)^{2}\right)}
\end{aligned}
$$

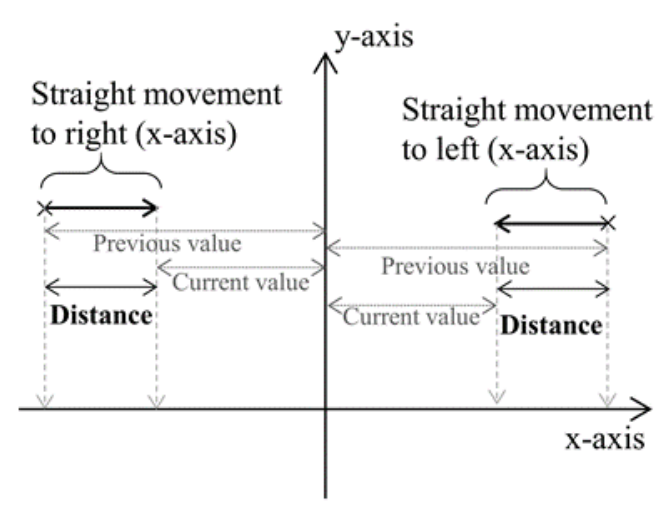

(a)Straight movements (left or right)

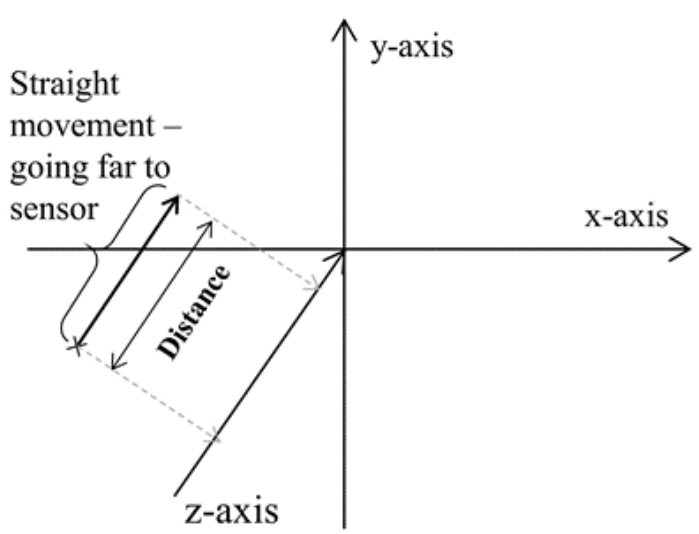

(c)Straight movements (coming close or going far)

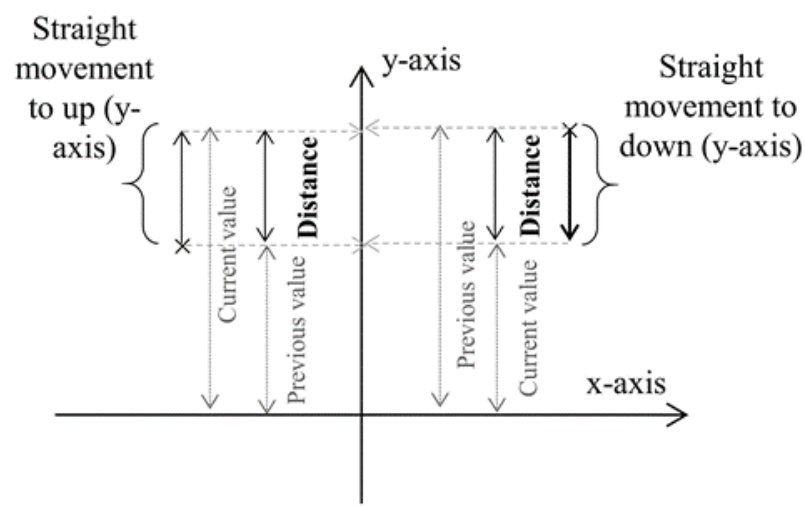

(b)Straight movements (up or down)

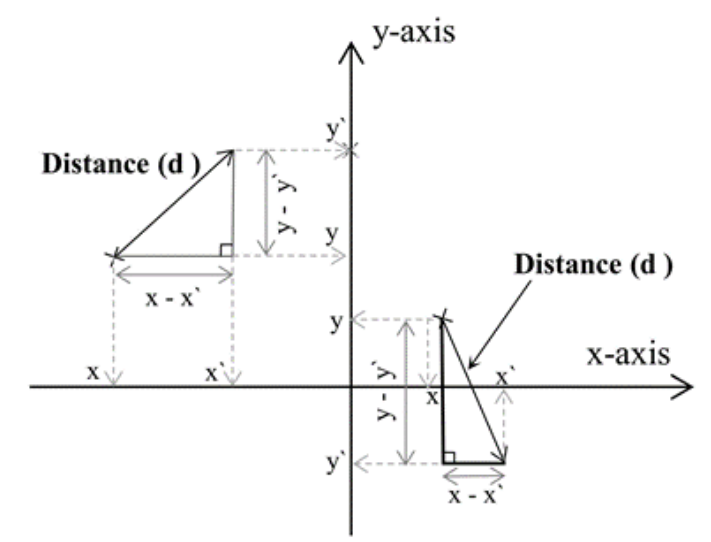

(d) Irregular distance on (x-axis and $\mathrm{y}$-axis)

Fig. 3 Coordinate system and velocity calculation description. 


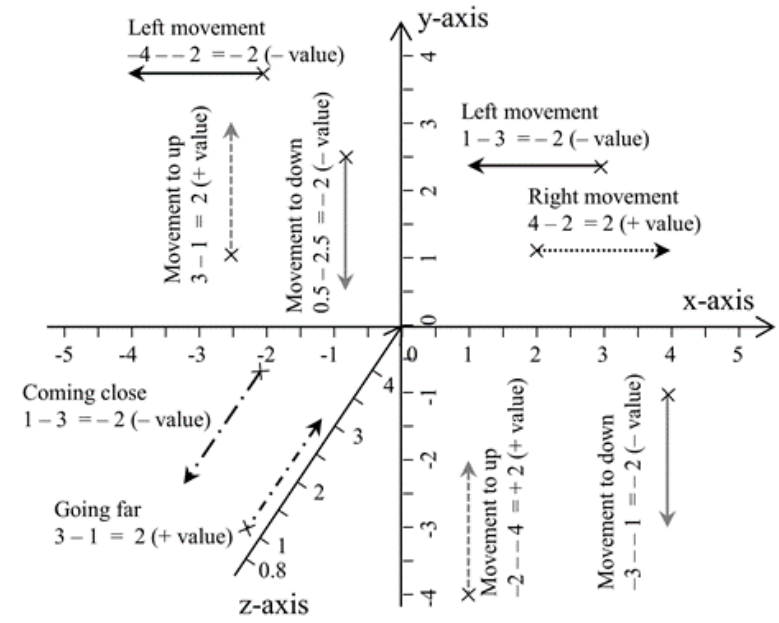

Fig. 4 Description of direction of movements.

The position of head, shoulder center, hip center, left and right knee are derived by considering the height of the particular joint and the joint coordinates ( $\mathrm{x}, \mathrm{y}$ and $\mathrm{z}$ values). Height of any joint is calculated by applying the joint coordinates to equation 2 . The height of the next joint and the angle formed by the joints are also considered in determining the current posture. An example of joint of angle calculation for the joint formed in between shoulder center, hip center and left knee while sitting on floor is illustrated in Fig. 5. with the equations. In this example the angle formed by the line joining shoulder center to hip center and left knee to hip center is calculated by dividing it into two parts. The first part labelled as 'a1' is calculated from the right-angle triangle formed by extending the $\mathrm{x}$-axis coordinate of hip center and y-axis coordinate of shoulder center. The angle 'a1' is calculated by taking the inverse tangent of $\mathrm{x}$-axis difference between hip center and shoulder center (opposite) and y-axis difference of the same two joints (adjacent). Similarly, angle 'a2' is calculated by taking the inverse tangent of $\mathrm{x}$-axis difference between hip center and left knee (opposite) and y-axis difference of the same two joints (adjacent). The final angle formed by three joints is the sum of the two angles $(\mathrm{a} 1+\mathrm{a} 2)$. The same concept is applied to calculate the angle between any three joints.

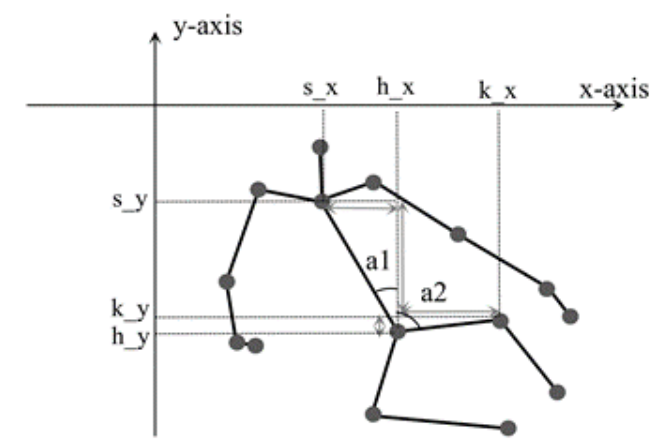

Fig. 5 Description of angles while sitting on floor.

$$
a 1=\tan ^{-1}\left(\frac{h_{-} x-S_{-} x}{S_{y} y-h_{y} y}\right)=\left(\frac{0.18864}{0.2705}\right)=34.89^{\circ}
$$

$$
a 2=\tan ^{-1}\left(\frac{k_{-} x-h_{-} x}{h_{-} y-k_{\_} y}\right)=\left(\frac{0.294}{0.0392}\right)=82.40^{\circ}
$$

\section{Results and discussion}

The proposed system has been tested with different activities of daily life such as walking, running, sitting on chair, sitting on floor, lying on floor and falling from standing. The experimental results showed that the proposed system can classify human fall from other activities of daily life using height changes and velocity of the subject together with the position of the subject after fall. The extraction of joint position after a potential fall activity showed good performance in differentiating human activities and confirming human fall.

The order of velocity and height of the subject used in the proposed algorithm greatly helped in eliminating human activities that are closely analogous to fall before going to the final stage for fall confirmation. Thus, the algorithm proved to reduce the error rate since those activities that are mostly misinterpreted as fall (such as lying on floor) is classified out in stage 3 (of Fig. 2.) before posture computation for confirming falls. Fig. 6 . shows screenshot of some activities performed in experimental testing and the corresponding changes of height of the subject are shown in Fig. 7. As seen from Fig. 7, the height changes pattern can distinguish some activities without the consideration velocity, but for activities that possess similar height change pattern does require velocity.

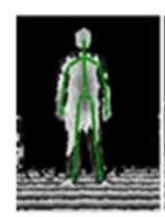

(a)

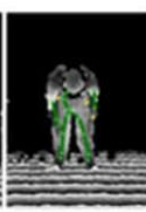

(b)

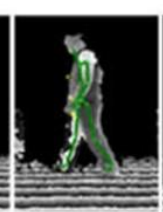

(c)

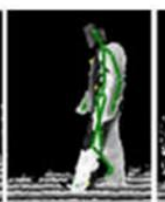

(d)

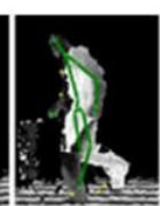

(e)

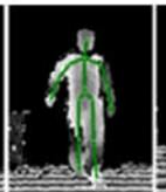

(f)

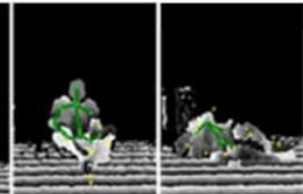

(g)

(h)

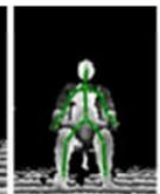

(i)

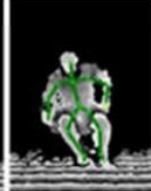

(j)

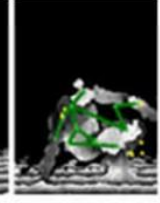

(k)

Fig. 6 Screenshot of activities performed with corresponding depth images. (a) Standing; (b) bend and stand up; (c) Walking across the sensor; (d) Walking slowly across the sensor; (e) Running; (f) Walking around the sensor; (g) sit down on floor; (h) falling from standing; (i) sit on chair; (j) fall from chair; (k) stand up. 


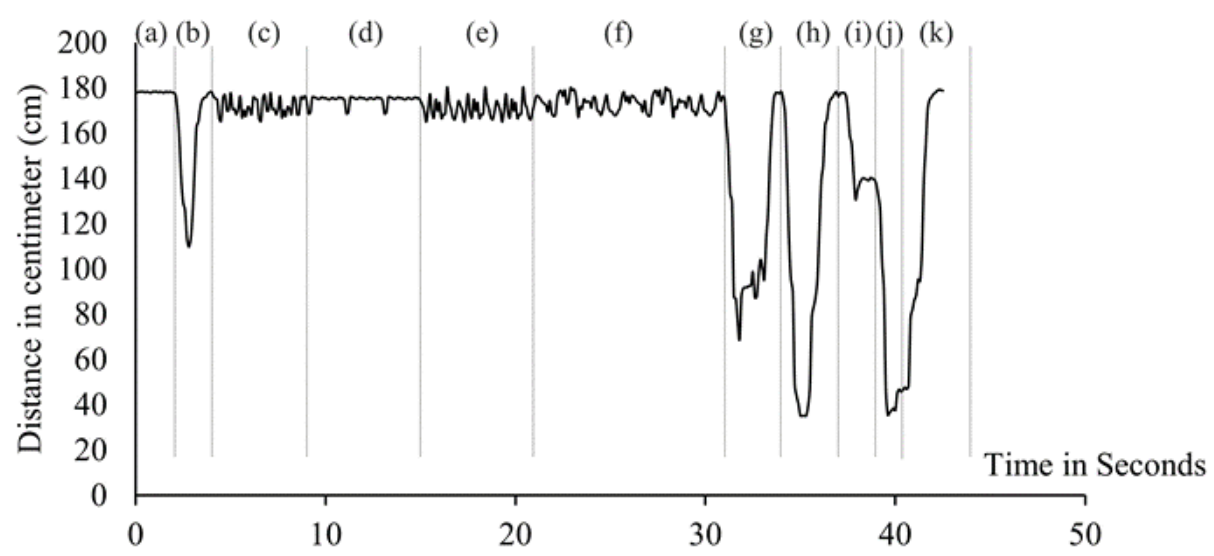

Fig. 7 Changes of height for the activities in Fig. 6.

The effectiveness of the algorithm to determine the direction of the movement is extremely important, because the variables used to calculate the parameter (used in fall detection) are dependent on the direction of the movement from previous location to new location. Therefore, an incorrect direction can lead to a calculation of a wrong parameter and thus it can bias the fall prediction. Two deliberate lying on floor from two sides to the viewing angle of sensor with almost the same duration are shown in Fig. 8. The pattern described in Fig. 8, legend (a) is while lying on floor in front (facing) of the sensor and legend (b) is showing the changes in height during lying on floor from a side of view of the sensor. Legend (c) shows the difference in the drop of height with mean of just 4.185 centimeter between the two lying pattern. It can be concluded from Fig. 8, that the system is accurate in determining the direction of movements, because for the two lying on floor in Fig. 8, the fall detection parameters must use different variables (two lying are on different view to sensor and thus the direction of height will be different). The similarity of height change for two lying, shows that the system had considered the direction of the movement correctly.

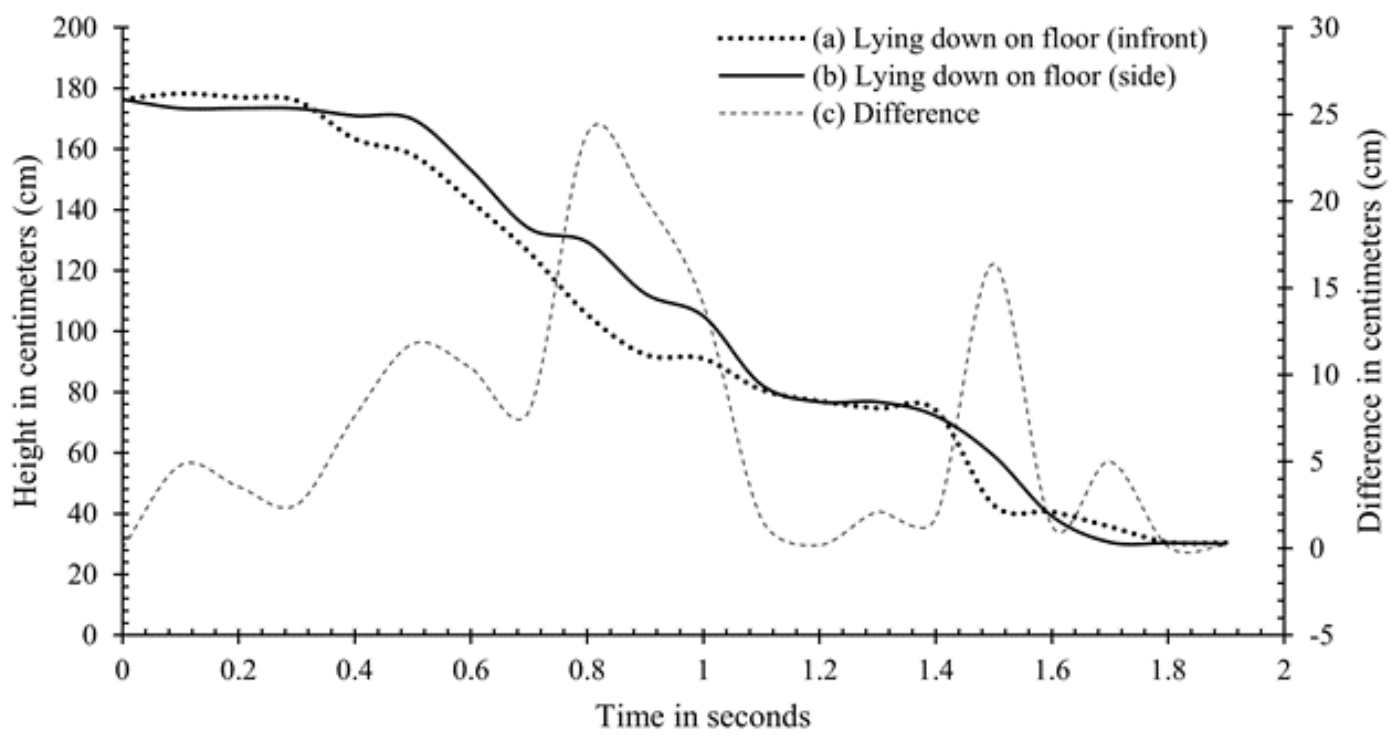

Fig. 8 Changes in height during lying on floor from different sides.

On the other hand, Fig. 9 illustrates the changes of height for walking across (horizontal) the sensor, around the view of the sensor, straight to the direction the sensor is pointed and diagonal to two sides the sensor is pointed. From the Fig. 9. and the statistics in Table 3, the system was able calculate height more accurately for walking straight to the direction the sensor is pointed. It can also be concluded that the accuracy dropped for movements across the sensor and movements diagonally to two sides where the sensor is pointed.
Table 3 Mean and Standard deviation for walking.

\begin{tabular}{lll}
\hline & Mean & STD \\
\hline Across the sensor view & 176.4152 & 8.239622 \\
Around the sensor view & 176.9429 & 7.523967 \\
Diagonally to sensor & 185.7137 & 8.095673 \\
Forth and back to sensor & 175.3316 & 4.645903 \\
\hline
\end{tabular}




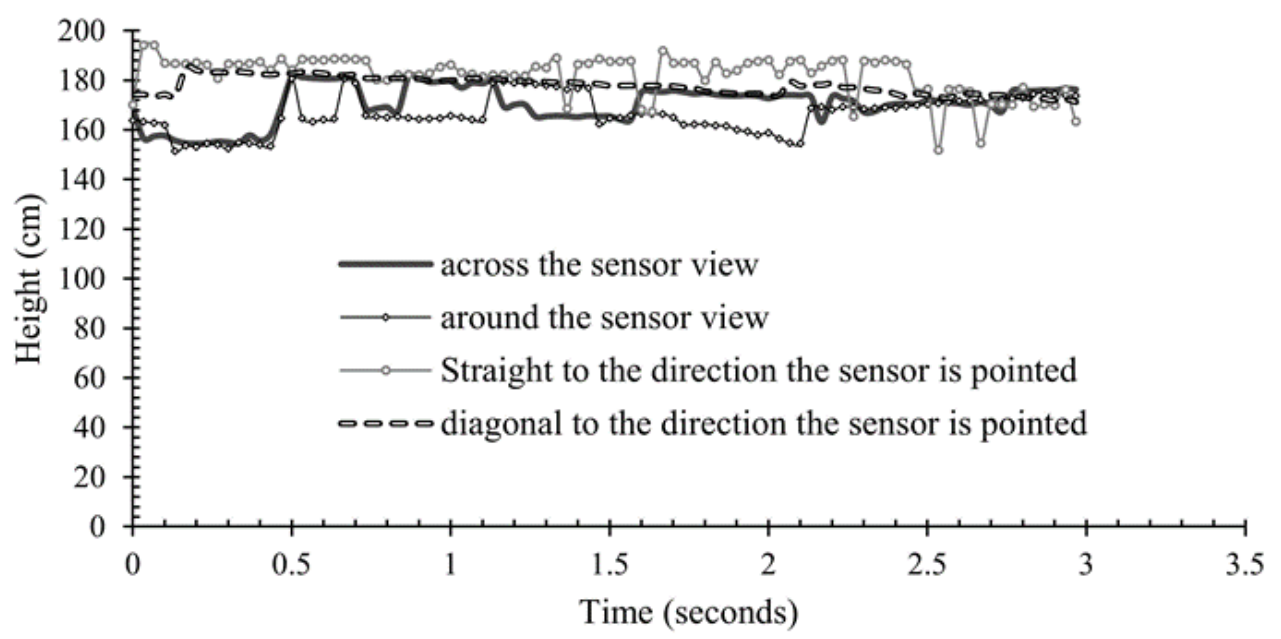

Fig. 9 Changes of height for walking to different sides on the viewing of the sensor.

The results presented so far is enough to prove that that proposed system is able to identify the direction of the movements accurately. The direction identification in vital, because the parameters used in the system is calculated based on the direction of the movement with respective to the previous location. In order to verify that the developed system can precisely predict any fall movement, it is important to prove that the parameters used in the fall detection process is calculated accurately. The Fig. 10, shows the calculated height changes during a normal slow lying on floor from standing together with head, shoulder center and hip position changes on y-axis of the image. It is clear from the Fig. 10. that the calculated height was very accurate as compared with the actual position data.

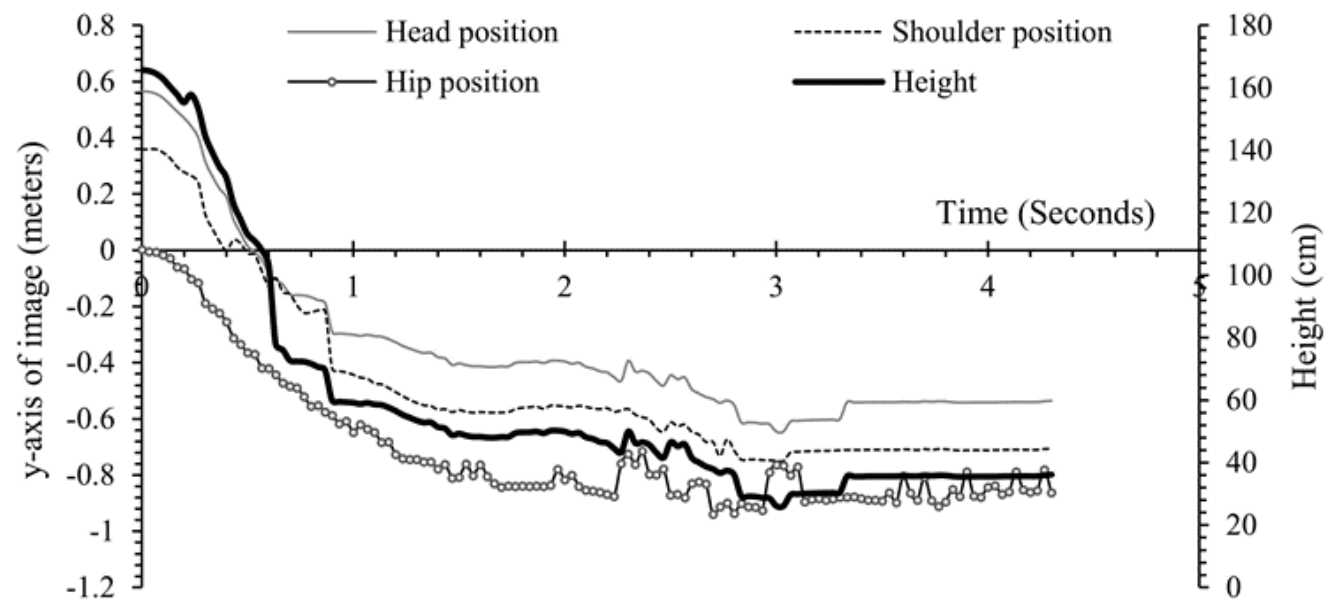

Fig. 10 Changes in height and position for lying on floor.

From the experimental results, it was found that the one daily activity which is very similar to fall is lying on floor. Fig. 11. illustrates the changes of height during normal slow lying on floor and falling on floor from standing together with their respective changes in velocity (downwards). The changes in height are similar in nature except the rate of change over the time. Falling on floor took about 2.1 seconds while lying on floor took nearly 3.5 second to completely rest on the floor. 


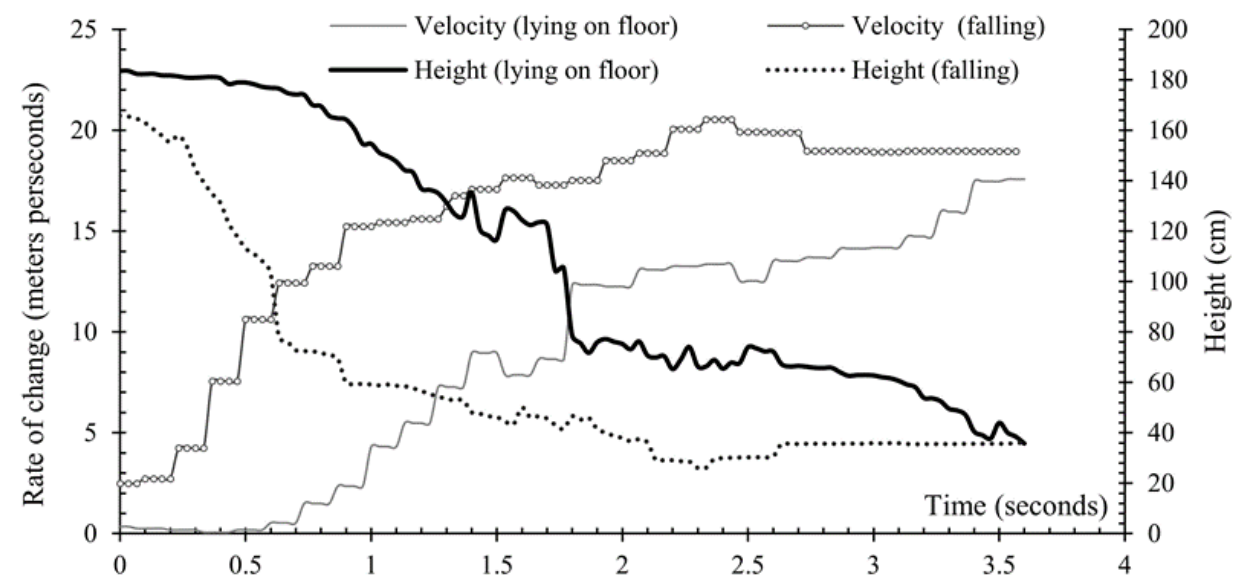

Fig. 11 Changes in height during fall and lying on floor with their velocity.

Table 4 shows the confusion matrix of falls and nonfalls from the experimental activities performed. It shows the overall number of trials conducted with the predicted value for each of the activity. Accuracy, sensitivity and specificity is calculated using the following equation 7,8 and 9 respectively.

$$
\begin{aligned}
& \text { Accuracy }==\frac{\mathrm{TP}+\mathrm{TN}}{\mathrm{TP}+\mathrm{TN}+\mathrm{FP}+\mathrm{FN}} \\
& \text { Sensitivity }==\frac{\mathrm{TP}}{\mathrm{TP}+\mathrm{FN}} \\
& \text { Specificity }=\frac{\mathrm{TN}}{\mathrm{FP}+\mathrm{TN}}
\end{aligned}
$$

\begin{tabular}{|c|c|c|c|}
\hline & \multirow[b]{2}{*}{ Total } & \multicolumn{2}{|c|}{ Predicted } \\
\hline & & Falls & None-falls \\
\hline \multirow{2}{*}{ 莺 } & Falls & $\begin{array}{c}\text { True Positive (TP) } \\
17\end{array}$ & $\begin{array}{c}\text { False Negative } \\
(\text { FN) } \\
0\end{array}$ \\
\hline & None-falls & $\begin{array}{c}\text { False Positive (FP) } \\
4\end{array}$ & $\begin{array}{c}\text { True Negative } \\
\text { (TN) } \\
56\end{array}$ \\
\hline
\end{tabular}

Table 4 The matrix for average of fall and non-fall values.

Using the above three equations on the average of fall and none-fall activities in Table 4, the system was able to gain an average accuracy of $94.81 \%$ with specificity of $93.33 \%$ and sensitivity of $100 \%$. The reason for the $100 \%$ sensitivity, is the fact that the developed system was able to classify all fall movements conducted in the trial. The reduced specificity is due to the inability of the system to identify some lying down on floor from fall.

\section{Conclusion}

This paper proposed a human fall detection system based on human height, velocity and position of body computed from depth images generated by the Microsoft Kinect sensor. The experimental results showed that the algorithm used on the system can accurately distinguish fall movements from other daily activities with an average accuracy of $94.81 \%$. The system was also able to gain a sensitivity of $100 \%$ with a specificity of $93.33 \%$. The proposed system was able to accurately distinguish all fall movements from other activities of daily life, even though it failed to identify some lying down on floor from fall. The velocity of joints greatly helps to classify certain movements where the distance change pattern possessed similar variation. The proposed system could be further improved by considering additional joints especially in the final stage for fall confirmation. Furthermore, experiments can be done to see if the velocity can be improved by increasing the gap for joint coordinate extraction.

\section{Acknowledgements}

This paper was partly sponsored by Center for Graduate Studies. This work is funded under the project titled "Biomechanics computational modeling using depth maps for improvement on gait analysis". The Author Yoosuf Nizam would also like to thank the Universiti Tun Hussein Onn Malaysia for providing lab components and GPPS (Project Vot No. U462) sponsor for his studies.

\section{References}

[1] Baker S.P. and Harvey A. Fall injuries in the elderly. Clinics in geriatric medicine, Vol 1(3), (1985), pp. 501-512.

[2] Griffiths C., Rooney C., and Brock A. Leading causes of death in England and Wales-how should we group causes. Health Statistics Quarterly, Vol 28(9), (2005), pp.

[3] Kannus P., Sievänen H., Palvanen M., Järvinen T., and Parkkari J. Prevention of falls and consequent injuries in elderly people. The Lancet, Vol 366(9500), (2005), pp. 1885-1893.

[4] Mohd M.N.H., Kashima M., Sato K., and Watanabe M. A Non-invasive Facial VisualInfrared Stereo Vision Based Measurement as an 
Alternative for Physiological Measurement. in (2015) Lecture Notes in Computer Science (including subseries Lecture Notes in Artificial Intelligence and Lecture Notes in Bioinformatics). (2014). Springer.

[5] Mohd M.N.H., Kashima M., Sato K., and Watanabe M. Internal state measurement from facial stereo thermal and visible sensors through SVM classification. ARPN Journal of Engineering and Applied Sciences, Vol 10(18), (2015), pp. 8363-8371.

[6] Tomari R., Kobayashi Y., and Kuno Y. Multiview head detection and tracking with long range capability for social navigation planning. in International Symposium on Visual Computing. (2011). Springer.

[7] Bian Z.-P., Chau L.-P., and Magnenat-Thalmann N. A depth video approach for fall detection based on human joints height and falling velocity. International Coference on Computer Animation and Social Agents, (2012), pp.

[8] Nizam Y., Jamil M.M.A., and Mohd M.N. A Depth Image Approach to Classify Daily Activities of Human Life for Fall Detection Based on Height and Velocity of the Subject. in International Conference on Movement, Health and Exercise. (2016). Springer.

[9] Nizam Y., Mohd M.N.H., and Jamil M.M.A. Classification of Human Fall from Activities of Daily Life using Joint Measurements. Journal of Telecommunication, Electronic and Computer Engineering (JTEC), Vol 8(4), (2016), pp. 145149.

[10] Nizam Y., Mohd M.N.H., and Jamil M.M.A. Human Fall Detection from Depth Images using Position and Velocity of Subject. Procedia Computer Science, Vol 105, (2017), pp. 131137.

[11] Mohd M.N.H., Nizam Y., Suhaila S., and Jamil M.M.A. An optimized low computational algorithm for human fall detection from depth images based on Support Vector Machine classification. (2017), pp.

[12] Nizam Y., Mohd M.N., and Jamil M.M.A. BIOMECHANICAL APPLICATION: EXPLOITATION OF KINECT SENSOR FOR GAIT ANALYSIS. (2006), pp.

[13] Kwolek B. and Kepski M. Human fall detection on embedded platform using depth maps and wireless accelerometer. Computer methods and programs in biomedicine, Vol 117(3), (2014), pp. 489-501.

[14] Kepski M. and Kwolek B. Fall detection using ceiling-mounted 3d depth camera. in Computer Vision Theory and Applications (VISAPP), 2014 International Conference on. (2014). IEEE.

[15] Zhang Z., Liu W., Metsis V., and Athitsos V. A viewpoint-independent statistical method for fall detection. in Pattern Recognition (ICPR), 2012 21 st International Conference on. (2012). IEEE.

[16] Yang L., Ren Y., Hu H., and Tian B. New fast fall detection method based on spatio-temporal context tracking of head by using depth images. Sensors, Vol 15(9), (2015), pp. 23004-23019.

[17] Kawatsu C., Li J., and Chung C., Development of a fall detection system with Microsoft Kinect, in Robot Intelligence Technology and Applications 2012. Springer, (2013), pp. 623630.

[18] Mundher Z.A. and Zhong J. A Real-Time Fall Detection System in Elderly Care Using Mobile Robot and Kinect Sensor. International Journal of Materials, Mechanics and Manufacturing, Vol 2(2), (2014), pp. 133-138.

[19] Rougier C., Auvinet E., Rousseau J., Mignotte M., and Meunier J., Fall detection from depth map video sequences, in Toward useful services for elderly and people with disabilities. Springer, (2011), pp. 121-128.

[20] Gasparrini S., Cippitelli E., Spinsante S., and Gambi E. A depth-based fall detection system using a Kinect® sensor. Sensors, Vol 14(2), (2014), pp. 2756-2775.

[21] Gasparrini S., Cippitelli E., Gambi E., Spinsante S., Wåhslén J., Orhan I., and Lindh T., Proposal and experimental evaluation of fall detection solution based on wearable and depth data fusion, in ICT Innovations 2015. Springer, (2016), pp. 99-108.

[22] Nizam Y., Mohd M.N.H., Tomari R., and Jamil M.M.A. Development of Human Fall Detection System using Joint Height, Joint Velocity, and Joint Position from Depth Maps. Journal of Telecommunication, Electronic and Computer Engineering (JTEC), Vol 8(6), (2016), pp. 125131. 